\title{
COMO OBTER NANOPARTÍCULAS DE OURO USANDO SUCO DE LIMÃO?
}

\author{
Mariana F. Coura, Guilherme Arroyos, Lilian Cherubin Correia e Regina C. G. Frem* \\ Departamento de Química Geral e Inorgânica, Instituto de Química de Araraquara, Universidade Estadual Paulista, 14800-060 \\ Araraquara - SP, Brasil
}

Recebido em 13/12/2017; aceito em 01/02/2018; publicado na web em 22/02/2018

\begin{abstract}
HOW TO OBTAIN GOLD NANOPARTICLES FROM LEMON JUICE? This article refers about a green method for the preparation of gold nanoparticles using juice from four types of lemon (Tahiti, Key lime, Sicilian and Rangpur). The approach is based on a classic synthesis of nanoparticles using sodium citrate as reducing agent, since all these lemon varieties have citric acid in their compositions. Several characterization techniques demonstrated the efficacy of the method for obtaining red colloidal solutions (nanoparticles in the order of 5-25 nm). The importance of this attempt to make environmentally friendly synthesis of gold nanoparticles is linked to the several applications of these nanomaterials, the most important being biomedicine, catalysis, electronics, biochemical sensing and imaging.
\end{abstract}

Keywords: gold nanoparticles; lemon; natural reducing agents; green synthesis.

\section{INTRODUÇÃO}

Nanopartículas são partículas que existem em uma escala nanométrica com tamanhos de até $100 \mathrm{~nm}$ em pelo menos uma dimensão. ${ }^{1,2}$ Materiais constituídos por pequenas partículas sempre foram de grande interesse para a humanidade. O conceito de átomo é conhecido desde a Antiguidade, em que era definido como uma partícula extremamente pequena (e até então, indivisível) formadora da matéria. ${ }^{3}$ Atualmente, sabe-se que os átomos possuem tamanho na ordem de $10^{-12}$ a $10^{-10} \mathrm{~m}$. Nestas escalas, devido aos efeitos quânticos, os materiais podem apresentar propriedades físicas e químicas únicas, tais como homogeneidade (tamanho e forma) de partícula, condutância térmica, resistência à oxidação, além de propriedades ópticas, elétricas e magnéticas especiais. Por essa razão, principalmente a partir do início da década de 2000, a nanotecnologia tem se incorporado em diversas áreas do conhecimento tais como tecnologia da informação, fotônica, plasmônica, biomedicina, energia, catálise, entre outras. ${ }^{4-12}$ No que se refere, por exemplo, a pesquisas de aplicação na área médica, incluindo tratamento via hipertermia de alguns tipos de câncer, ${ }^{13}$ a intenção é tornar os procedimentos mais baratos, mais fáceis de serem administrados e também mais seguros. Sob esse ponto de vista, têm surgido importantes trabalhos quanto a avaliação da toxicidade destes materiais em escala nanométrica, levando ao desenvolvimento da área chamada nanotoxicologia. ${ }^{14,15}$

Dentro do universo das partículas nanometálicas, as nanopartículas de ouro foram as primeiras a serem descobertas, provavelmente pelo fato de poderem ser preparadas com muita facilidade. O ouro é um metal de grande importância histórica devido ao seu alto valor agregado e sua beleza associada. Além disso, devido a suas propriedades químicas de metal nobre, é utilizado em eletrônica e na área médica (em próteses, por exemplo). Em sua forma nanométrica, o ouro coloidal apresenta uma coloração vermelha/violeta, propriedade essa que foi utilizada na Roma Antiga para a confecção de vidros. ${ }^{16}$ Pesquisas recentes envolvendo nanopartículas de ouro buscam aplicá-las principalmente na área médica, devido à sua excelente biocompatibilidade. Além de ser relativamente inerte, o ouro nanoparticulado apresenta propriedades características de nanomateriais, tais como elevada razão área superficial por volume de solução, propriedades

*e-mail: rcgfrem@iq.unesp.br ópticas e eletrônicas únicas e facilidade de modificação de sua superfície. ${ }^{17}$ Além disso, o diâmetro reduzido desses materiais facilita a sua entrada no interior das células, possibilitando uma série de diagnósticos, imageamentos e tratamentos intracelulares. ${ }^{18}$ Diversos compostos podem ser incorporados às partículas de ouro agregando propriedades específicas destes compostos no material. ${ }^{19}$ Por essa razão, nanopartículas de ouro, modificadas ou não, possuem aplicação em diversas áreas como óptica, eletrônica, magnética e catálise. ${ }^{20-25}$

Em geral, há duas estratégias distintas para a obtenção de nanomateriais, top-down e bottom-up. Os métodos top-down consistem na remoção de matéria de estruturas originalmente maiores (material bulk) para obter a nanoestrutura desejada. Por outro lado, no princípio bottom-up, os componentes do material presentes inicialmente em escala atômica, se auto organizam nos materiais nanoestruturados. ${ }^{26}$ Particularmente no que diz respeito a preparação de nanopartículas de ouro, as técnicas bottom-up são mais usuais, englobando diversos métodos físicos (uso de ultrassom de alta frequência, por exemplo) ${ }^{27}$ e químicos. ${ }^{28,29}$ No entanto, o tamanho, a morfologia, a estabilidade e a funcionalidade da superfície das nanopartículas dependem não só do método de síntese empregado como também das condições experimentais (concentração do íon metálico, velocidade de redução, temperatura, uso e concentração de agentes estabilizantes, etc.). ${ }^{30-32}$ Entre os métodos químicos, a maioria usa o ácido cloroáurico, $\left[\mathrm{HAuCl}_{4}\right]$, como fonte de íons $\mathrm{Au}(\mathrm{III})$ e diversos agentes redutores como citrato de sódio (método de Turkevich), ${ }^{33}$ borohidreto de sódio (métodos de Brust e de Martin), ${ }^{34,35}$ hidroquinona, ${ }^{36}$ entre outros. ${ }^{37,38}$ Nanopartículas de ouro também podem ser formadas pela redução química dos íons metálicos no interior de micelas (ou micelas reversas) formadas por surfactantes e usadas como nanoreatores. Adicionalmente, na síntese micelar, o surfactante também auxilia na estabilização das nanopartículas formadas. ${ }^{39}$

Como grande parte das aplicações das nanopartículas de ouro concentra-se na área biomédica, é imprescindível que haja um conhecimento e um controle da sua toxicidade no organismo humano. ${ }^{40}$ Enquanto a nanotoxicologia trata do potencial dano causado pelas nanopartículas, os métodos químicos para a sua preparação podem deixar alguns resíduos tóxicos no meio reacional ou adsorvidos na superfície. O precursor ácido cloroáurico, por exemplo, é um ácido extremamente forte e corrosivo, sendo então necessário que ele seja consumido por completo. No que diz respeito aos agentes redutores 
comumente usados, o borohidreto de sódio é um redutor muito forte e extremamente tóxico para as células. A hidroquinona, por sua vez, é um composto já utilizado na medicina como clareador de pele, mas, no entanto, é suspeita de ser carcinogênica. ${ }^{41}$ Dentro desse contexto, o método de Turkevich seria o mais recomendado, ${ }^{42}$ visto que o citrato de sódio não é tóxico e é até mesmo utilizado como aditivo em alimentos.

Visando o uso de técnicas de preparação ambientalmente compatíveis, ${ }^{43}$ nanopartículas de ouro têm sido obtidas via biossíntese usando materiais naturais como extrato de plantas, microrganismos (bactérias, fungos, algas) e biomoléculas (enzimas, por exemplo). ${ }^{44-51} \mathrm{E}$ sob essa perspectiva, surgiu então a ideia de utilizar extratos de limão (gênero Citrus) e suas variedades por serem ricos em ácido cítrico (por essa razão, compõem a família das chamadas frutas cítricas). Sendo assim, a proposta desse trabalho consistiu em utilizar o suco dessas frutas na síntese de nanopartículas de ouro, em substituição ao citrato de sódio usado por Turkevich. As espécies de limão mais conhecidas no Brasil são limão taiti (Citrus $\times$ latifolia), limão siciliano (Citrus $\times$ limon), limão cravo (Citrus $\times$ limonia) e limão galego (Citrus $\times$ aurantiifolia). Uma das vantagens em utilizá-las na síntese das nanopartículas é o seu baixo custo e alta disponibilidade, sendo que todas as variedades são amplamente cultivadas no Brasil, dependendo das características da região e da época do ano.

\section{PARTE EXPERIMENTAL}

Baseando-se nos princípios da química verde, foi desenvolvido nesse trabalho um método para sintetizar nanopartículas de ouro usando o ácido cítrico presente no limão como redutor de íons $\mathrm{Au}(\mathrm{III})$. Foi investigada também a influência da concentração dos reagentes e da natureza do composto metálico de partida no tamanho das nanopartículas obtidas. Além disso, os diferentes tipos de limão foram empregados para observar se haveria alguma diferença nas nanopartículas de ouro obtidas e a quantidade de ácido cítrico em cada um dos sucos foi determinada por cromatografia líquida de alta eficiência (CLAE-DAD). Em todos os casos, toda a vidraria foi lavada com água régia $\left(1 \mathrm{HNO}_{3}: 3 \mathrm{HCl}\right)$, a fim de garantir que possíveis impurezas fossem removidas (procedimento realizado por 10 minutos, na capela do laboratório). Em seguida, retirou-se a solução de água régia e lavou-se várias vezes essas vidarias com água destilada.

\section{Preparo das soluções redutoras}

O suco dos limões (taiti, siciliano, cravo e galego) foi extraído e peneirado, utilizando uma peneira de cozinha, para separação das sementes e polpa. Após extração e peneiramento dos sucos dos limões, as diferentes suspensões obtidas foram colocadas em eppendorfs e centrifugadas por 10 minutos a $13000 \mathrm{rpm}$. Com o auxílio de uma pipeta de Pasteur, separou-se cuidadosamente o sobrenadante do filtrado. Os sobrenadantes foram imediatamente submetidos à filtração a vácuo, seguida pelo ajuste do $\mathrm{pH}$ de $\sim 2,2$ para 6,2 - 8,0, utilizando $\mathrm{NaOH} 1,0 \mathrm{~mol} \mathrm{~L}^{-1}$.

\section{Utilizando excesso de precursor metálico}

Foram separados quatro erlenmeyers de $125 \mathrm{~mL}$, dentro dos quais foi colocado $50,0 \mathrm{~mL}$ de uma solução de ácido cloroáurico de concentração $1,0 \times 10^{-3} \mathrm{~mol} \mathrm{~L}^{-1}$. O sistema foi aquecido a aproximadamente $100{ }^{\circ} \mathrm{C}$, sob agitação. Em seguida, após decorridos 5 minutos, desligou-se o aquecimento mantendo-se apenas a agitação e adicionou-se $10,0 \mathrm{~mL}$ do extrato dos limões, um em cada erlenmeyer distinto. Aproximadamente um minuto após a adição do suco, observou-se alteração de cor, passando de amarelo claro (cor original da solução de $\mathrm{HAuCl}_{4}$ ) para vermelho escuro. Após 5 minutos, desligou-se a agitação e observou-se as soluções contra a luz para verificar se as mesmas ainda eram translúcidas. Para posterior confirmação, incidiu-se um laser de cor verde através das nanopartículas para verificar se era possível observar sua trajetória através da amostra (efeito Tyndall), confirmando que a substância sintetizada era um coloide. Esses nanomateriais metálicos foram armazenadas sob baixa temperatura, para aumentar o seu tempo de duração. As nanopartículas de ouro assim preparadas foram caracterizadas por espectroscopia de absorção na região UV-Vis e microscopia eletrônica.

\section{Utilizando excesso de redutor}

Para realizar a síntese das nanopartículas de ouro, com o auxílio de uma pipeta graduada, colocou-se $2,0 \mathrm{~mL}$ de uma solução de $\mathrm{HAuCl}_{4}$ aproximadamente $10^{-3} \mathrm{~mol} \mathrm{~L}^{-1}$ em um béquer de $10 \mathrm{~mL}$. O sistema foi agitado e aquecido até uma temperatura de $100^{\circ} \mathrm{C}$, por 5 minutos. Desligou-se o aquecimento, mantendo-se a agitação, e adicionou-se $5,0 \mathrm{~mL}$ do suco dos limões. Foi observada rapidamente uma mudança na coloração da solução, passando de amarelada, característica da solução de ácido cloroáurico, para avermelhada, confirmando a obtenção das partículas metálicas em escala nanométrica. Manteve-se a reação por aproximadamente 10 minutos. Após o término de cada reação, as soluções coloidais contendo as nanopartículas de ouro foram armazenadas sob refrigeração.

\section{Usando ouro metálico como precursor}

Nesse experimento, partiu-se de uma pequena placa de ouro metálico $(0,2444 \mathrm{~g})$ para a produção do ácido cloroáurico. O metal foi dissolvido aos poucos em $\mathrm{HCl}$ concentrado (2,68 mL) e $\mathrm{HNO}_{3}$ concentrado $(0,465 \mathrm{~mL})$, a $90{ }^{\circ} \mathrm{C}$, em uma capela para a eliminação dos gases tóxicos. A dissolução completa da placa foi observada após 45 minutos de reação, obtendo-se uma solução concentrada (de cor alaranjada) de $\mathrm{HAuCl}_{4}$ como produto final. Através da estequiometria da reação representada na equação (1), foi possível estimar a concentração da solução de $\mathrm{HAuCl}_{4}$ obtida a partir da massa inicial de ouro metálico e do volume final presente no béquer.

$$
\mathrm{Au}^{0}+\mathrm{HNO}_{3}+4 \mathrm{HCl} \rightarrow \mathrm{HAuCl}_{4} \cdot 2 \mathrm{H}_{2} \mathrm{O}+\mathrm{NO}
$$

Diluiu-se, então, a solução precursora obtida até alcançar a concentração de $1,0 \times 10^{-3} \mathrm{~mol} \mathrm{~L}^{-1}$ e realizou-se a síntese das nanopartículas de ouro usando excesso do redutor químico (vide procedimento anterior) e o limão taiti, devido a sua maior disponibilidade. Novamente, observou-se uma alteração praticamente instantânea na cor da solução de amarelo para vermelho intenso.

\section{Quantificação do ácido cítrico}

Sucos dos quatro diferentes tipos de limão (galego, cravo, siciliano e taiti) foram extraídos, peneirados, centrifugados (10 min, $13000 \mathrm{rpm}$ ) e filtrados. Os sobrenadantes foram, então, diluídos em água ultrapura (Millipore, USA) na proporção de 1:20. O método de padronização externa foi realizado com padrão de referência de ácido cítrico (Supelco, Sigma Aldrich) a partir de diluições sucessivas de uma solução estoque com análises cromatográficas em triplicata nos pontos 2000, 1000, 800, 600, 400, $200 \mu \mathrm{g} \mathrm{mL}^{-1}$. Tanto a curva de calibração quanto as amostras dos limões foram analisadas em um cromatógrafo a líquido de alta eficiência com detector de arranjo de diodos (Ultimate 3000 RS, Dionex), em uma coluna analítica Synergi Hydro-RP C18 (150 x 4,6 mm, 4 mm, $80 \AA$ A, Phenomenex). As condições cromatográficas foram: modo de eluição isocrático com tampão 
fosfato (fosfato de sódio monobásico e ácido fosfórico, $\mathrm{pH}=2,6$ ) durante 10 minutos, $25^{\circ} \mathrm{C}$, temperatura de forno, $1,0 \mathrm{~mL} \mathrm{~min}^{-1}$ de vazão e $10 \mu \mathrm{L}$ de volume de injeção. Todas as detecções foram realizadas no comprimento de onda de $210 \mathrm{~nm}$.

\section{RESULTADOS E DISCUSSÃO}

\section{Utilizando excesso de precursor metálico}

Através do método apresentado, foram obtidas soluções contendo nanopartículas de ouro, caracterizadas por espectroscopia de absorção no UV-Vis e microscopia eletrônica de varredura. A Figura 1 ilustra os espectros eletrônicos, onde pode-se observar uma similaridade entre os perfis de absorção registrados para as nanopartículas de ouro sintetizadas com as quatro variedades distintas de limão. Como pode ser observado na Figura 1, o máximo de absorção de luz ocorre na região verde do espectro visível $(\sim 520 \mathrm{~nm})$, enquanto luz vermelha $(\sim 700 \mathrm{~nm})$ é refletida.

Essa banda de absorção na região de $520 \mathrm{~nm}$ indica a formação de nanopartículas de ouro devido à excitação das vibrações de plasma de superfície e é característica de partículas de ouro monodispersas com tamanho entre 5 e $30 \mathrm{~nm} .{ }^{52}$ Este fenômeno é conhecido como localized surface plasmon resonance (LSPR) e pode ser definido como a oscilação ressonante de elétrons livres na superfície de uma nanopartícula metálica em resposta ao campo elétrico oscilante da radiação eletromagnética. ${ }^{12}$

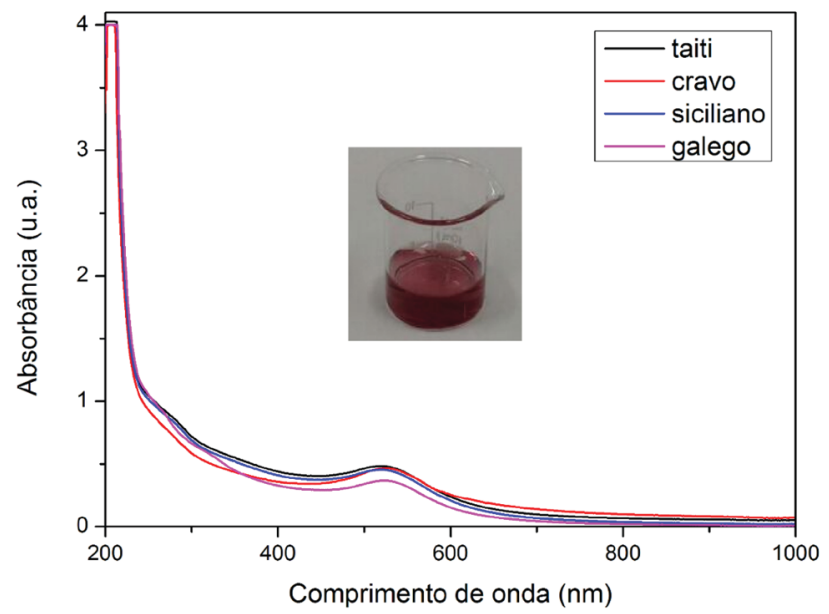

Figura 1. Espectros de absorção UV-Vis das nanopartículas sintetizadas com os quatro tipos de limão usando excesso de precursor metálico. Uma fotografia de uma solução vermelha de nanopartículas de ouro foi inserida a título de ilustração

Imagens de microscopia eletrônica de varredura foram obtidas para as nanopartículas de ouro sintetizadas com o limão taiti e estão ilustradas na Figura 2. Observou-se uma considerável homogeneidade de forma (quase-esférica) e tamanho (19,9 a 23,4 nm) das partículas sintetizadas quando excesso do precursor metálico foi usado.

Duas questões importantes podem ser levantadas nesse momento. A primeira delas é que o ânion citrato presente em quantidades similares ( $5 \%$ do suco) nos quatro tipos de limão usados neste trabalho deve ser o responsável pela redução dos íons Au(III). De fato, apesar de os quatro tipos de limão apresentarem teores diferentes de ácido ascórbico (aproximadamente entre 57,52 e 34,50 mg/100 mL do suco $)^{53}$ que também possui atividade antioxidante comprovada, ${ }^{54}$ isso não afetou na obtenção das nanopartículas metálicas, o que leva à conclusão que o agente redutor deve tratar-se realmente do ácido cítrico. Além disso, como essa rota sintética utilizou uma menor

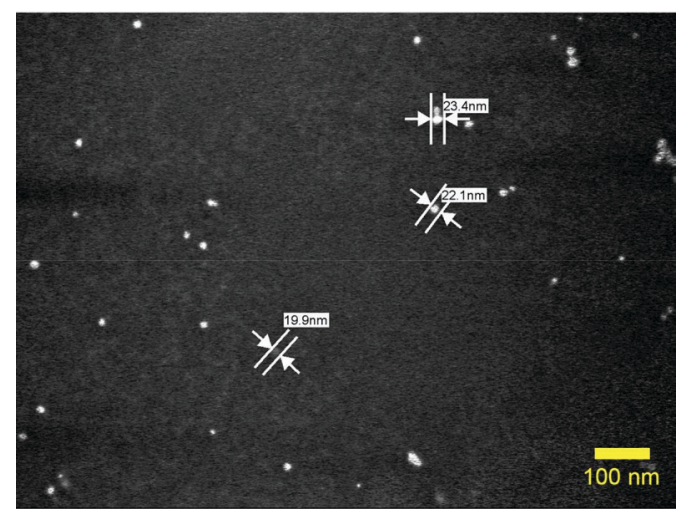

Figura 2. Imagem MEV-FEG das nanopartículas de ouro sintetizadas com o limão taiti usando excesso de precursor metálico

quantidade desse redutor, foram produzidas partículas maiores em relação àquelas obtidas usando as duas outras rotas sintéticas (ver a seguir).

\section{Utilizando excesso de redutor}

Segundo Kimling et al., ${ }^{55}$ altas concentrações de citrato estabilizam mais rapidamente nanopartículas de tamanhos menores. Dentro desse contexto, foi então investigada a influência do aumento na quantidade de extrato de limão no tamanho das partículas formadas. De fato, como ilustram as Figuras 3a-d, a microscopia eletrônica de varredura realizada para as amostras obtidas com as variedades taiti, cravo, siciliano e galego, respectivamente, confirmou a formação, nesse caso, de partículas menores (da ordem de 5-10 nm) quando comparadas àquelas obtidas com excesso de precursor metálico (ver Figura 2).

Para a amostra obtida a partir de excesso de limão taiti, imagens de microscopia eletrônica de transmissão também foram obtidas e encontram-se ilustradas na Figura 4.

Como pode ser visto na Figura 4, o método conduziu a formação de partículas com boa homogeneidade de forma e tamanho. Além disso, com uma maior aproximação, foi possível observar até mesmo alguns planos cristalográficos do retículo cristalino cúbico de face centrada associados ao ouro metálico..$^{56}$

Visando calcular o rendimento da síntese bem como comprovar que se tratava de fato de uma condição reacional na qual excesso de agente redutor estava presente, análises de ouro foram realizadas via espectrofotometria de absorção atômica (ContrAA 300 da AnalytikJena). Considerando o fato de que nosso grupo de pesquisa atua na área de Química de Coordenação, com ênfase nas MetalOrganic Frameworks (MOFs) ${ }^{57}$ e a grande dificuldade em separar as nanopartículas da solução via centrifugação, o material poroso ZIF-8 $^{58}$ foi usado com sucesso para esse fim. Desse modo, foi possível determinar a quantidade de íons $\mathrm{Au}(\mathrm{III})$ restantes na solução (1,75 $\mathrm{x} 10^{-6} \mathrm{~mol} \mathrm{~L}^{-1}$ ). Sabendo que a quantidade de íons $\mathrm{Au}(\mathrm{III})$ presentes originalmente na solução de partida era de $1,47 \times 10^{-3} \mathrm{~mol} \mathrm{~L}^{-1}$, uma eficiência de redução de $99,9 \%$ foi alcançada, o que confirma de fato que o redutor está em excesso nesse caso.

Portanto, embora o tamanho das nanopartículas esteja mais diretamente relacionado a processos de nucleação/crescimento, a quantidade do estabilizante citrato (agente redutor) afetou o tamanho das partículas de ouro, tendo sido formadas partículas menores quando uma maior quantidade de suco foi usada. ${ }^{29}$

A Figura 5 ilustra os espectros eletrônicos das soluções coloidais obtidas a partir da interação de íons Au(III) com excesso de redutor preparado a partir das quatro variedades de limão.

Como pode ser notado na Figura 5, a presença das nanopartículas 

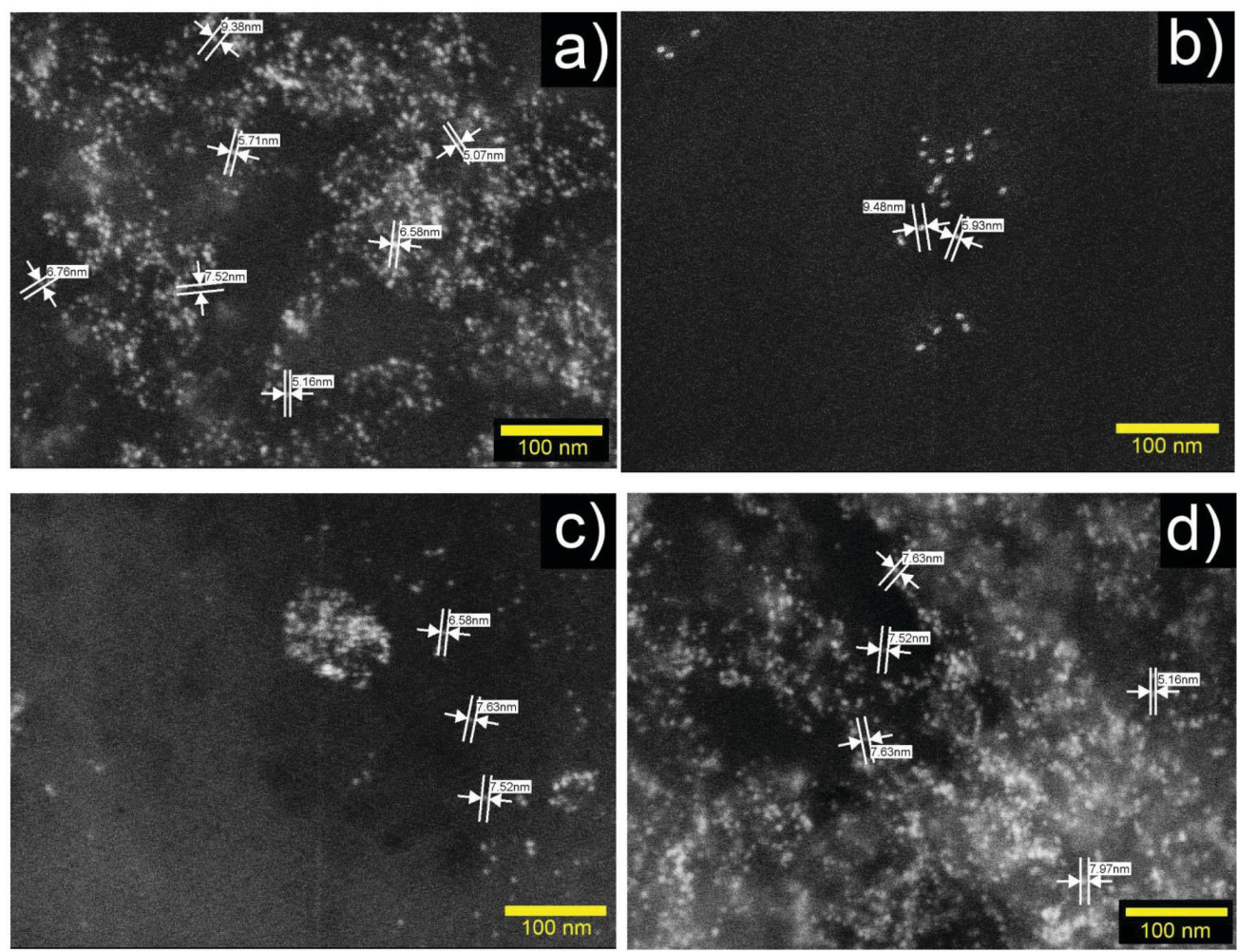

Figura 3. Imagem MEV-FEG das AuNPs obtidas com excesso de a) limão taiti; b) limão cravo; c) limão siciliano; d) limão galego
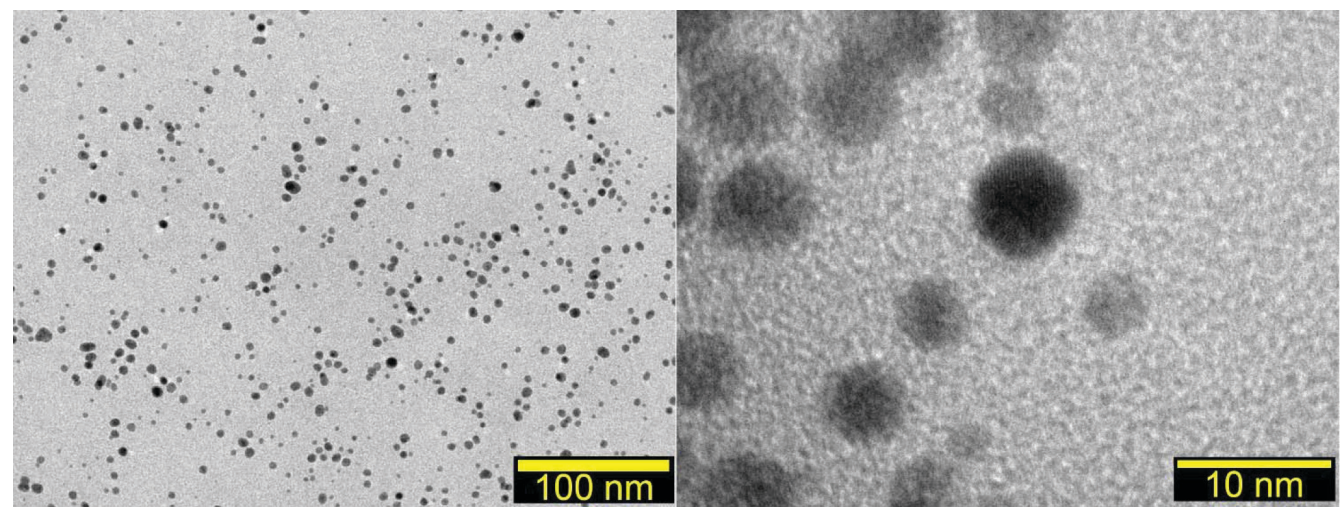

Figura 4. Imagens TEM das AuNPs obtidas com excesso de limão taiti

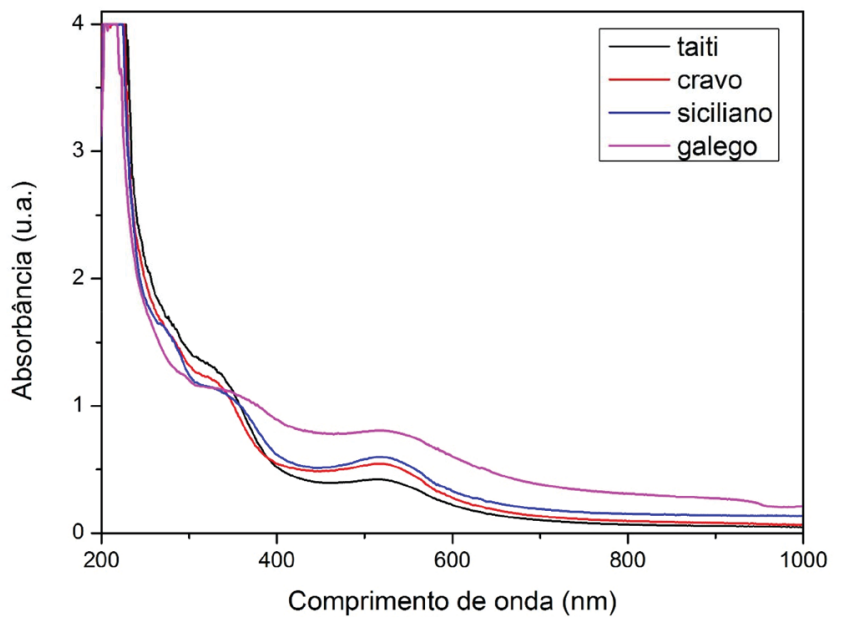

Figura 5. Espectros de absorção UV-Vis das nanopartículas sintetizadas com os quatro tipos de limão usando excesso de redutor metálicas pode ser comprovada pela banda larga cujo máximo de absorção encontra-se aproximadamente em $518 \mathrm{~nm}$ em todos os casos, característico de nanopartículas entre 5 e $10 \mathrm{~nm}$, em concordância com os resultados de microscopia. ${ }^{52}$ No entanto, quando se analisa os espectros na região do UV, alguns deslocamentos referentes ao suco cítrico, presente em maior quantidade nesse caso, são observadas. Por essa razão, espectros eletrônicos dos extratos foram obtidos e estão apresentados na Figura 6. De fato, os diferentes tipos de limão contêm diversos compostos orgânicos cujos grupos cromóforos absorvem nessa região do espectro eletromagnético. ${ }^{59}$ A Tabela 1 resume os dados espectroscópicos das amostras de limão, antes e após a adição do precursor de ouro.

\section{Usando ouro metálico como precursor}

Devido ao alto custo e baixa disponibilidade do precursor metálico utilizado $\left(\mathrm{HAuCl}_{4}\right)$, desenvolveu-se nessa etapa do trabalho uma metodologia alternativa que envolveu o uso de ouro metálico para a síntese das nanopartículas. Sendo assim, ácido cloroáurico foi 


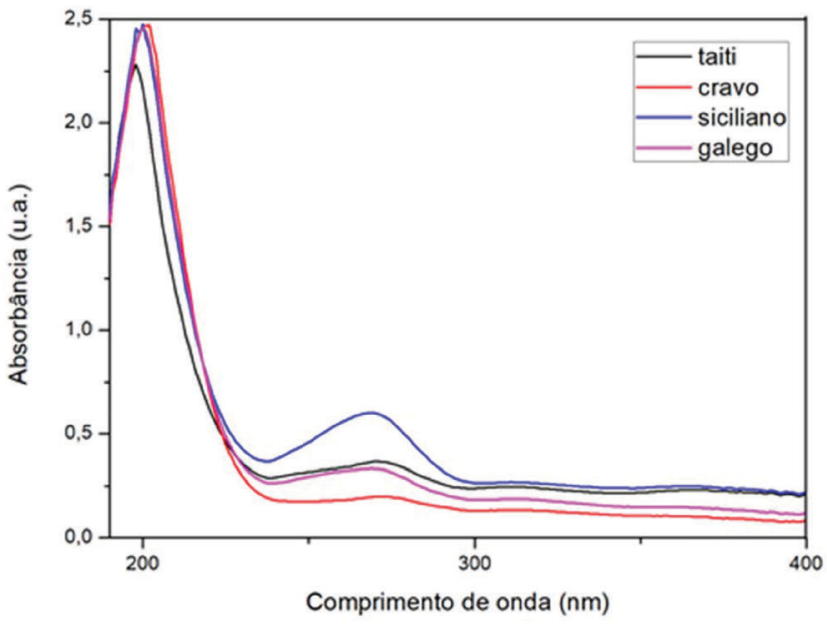

Figura 6. Espectros de absorção UV-Vis dos extratos dos quatro tipos de limão

Tabela 1. Dados espectroscópicos das amostras de limão, antes e após a adição do precursor de $\mathrm{Au}(\mathrm{III})$

\begin{tabular}{lcccc}
\hline Substância & \multicolumn{4}{c}{ Comprimento de onda (nm) } \\
\cline { 2 - 5 } & Taiti & Cravo & Siciliano & Galego \\
\hline Agente & 200 & 204 & 200 & 200 \\
Redutor & 270 & 270 & 268 & 270 \\
(limão) & 310 & 315 & 314 & 311 \\
& 364 & 361 & 363 & 364 \\
\hline Solução & 200 & 200 & 202 & 202 \\
coloidal de & - & - & 267 & 294 \\
AuNPs & 310 & 316 & 315 & 315 \\
& 518 & 519 & 518 & 518 \\
\hline
\end{tabular}

produzido (ver Figura 7) a partir da dissolução de uma pequena argola de uma corrente fina de ouro por uma mistura de ácidos clorídrico e nítrico. A Figura 7 também mostra o espectro eletrônico das nanopartículas sintetizadas, usando excesso de limão taiti.

Como ilustrado na Figura 7, além das bandas correspondentes a absorções dos componentes do limão (201 e $364 \mathrm{~nm}$ ), pode ser observada uma banda no visível (522 nm), exatamente na mesma

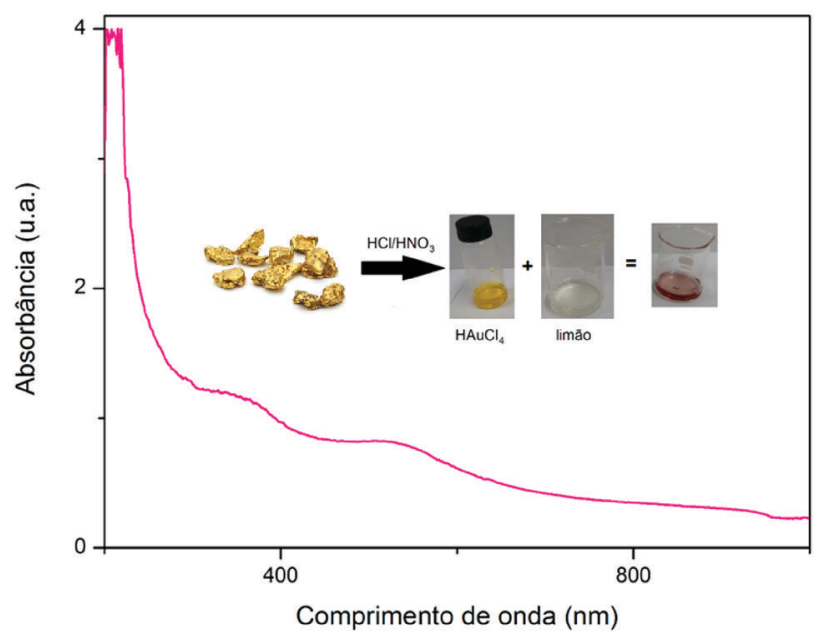

Figura 7. Espectro de absorção UV-Vis das nanopartículas sintetizadas a partir de ouro metálico usando excesso de limão taiti região onde aparece a absorção do material preparado com ácido cloroáurico.

Cabe destacar que em todos os experimentos realizados nesse trabalho, o $\mathrm{pH}$ foi ajustado para valores entre 6,2-8,0. Sabendo que os valores de $\mathrm{pKa}$ do ácido cítrico são, respectivamente, $\mathrm{pKa}_{1}=3,1$; $\mathrm{pKa}_{2}=4,5$ e $\mathrm{pKa}_{3}=5,8,{ }^{60}$ pode-se afirmar que ele está presente no meio reacional na forma do ânion citrato, atuando, portanto, como agente redutor na produção das nanopartículas vermelhas de ouro metálico. Esse resultado é importante tanto do ponto de vista econômico quanto ambiental, uma vez que houve uma redução considerável no consumo de hidróxido de sódio, tendo sido possível então a obtenção das AuNPs no $\mathrm{pH}$ próximo ao do solvente utilizado (água).

\section{Quantificação do ácido cítrico}

A avaliação da curva analítica foi realizada de acordo com algumas diretrizes da RDC No 166/2017 (ANVISA), ${ }^{61}$ sendo possível assegurar que figuras de mérito como linearidade, seletividade, limite de detecção $(\mathrm{LD}=3 \mathrm{~S} / \mathrm{R})$, limite de quantificação $(\mathrm{LOQ}=10 \mathrm{~S} / \mathrm{R})$ e precisão fossem avaliadas. Para linearidade, a análise da variância (ANOVA) e do coeficiente de correlação $(\mathrm{R}=0,99)$ a um nível de significância de $5 \%$ permitiram a constatação da homocedasticidade da regressão. A precisão em nível de repetitividade apresentou porcentagem do coeficiente de variação (\% CV) inferior a 4 para todas as réplicas da curva analítica.

Na Figura 8, é possível observar os cromatogramas obtidos para os quatro tipos de limão, nos quais pode-se notar uma composição muito semelhante entre os metabólitos constituintes dos diferentes limões, diferindo apenas na intensidade do sinal no tempo de retenção do ácido cítrico $\left(\mathrm{t}_{\mathrm{R}}=5,5 \mathrm{~min}\right)$.

Com a regressão linear construída $(y=0,015 x+0,116$, em que a variável x é referente à área do pico do ácido cítrico e y é concentração), foi possível estimar a concentração desse ácido em cada tipo de limão pela área adquirida nos cromatogramas. Portanto, as concentrações obtidas de ácido cítrico nos limões estão indicadas na Tabela 2.

\section{CONCLUSÃO}

Pelo fato de nanopartículas de ouro serem úteis para uma gama de aplicações, seria muito importante que fosse desenvolvido um método simples para produzi-las em um curto intervalo de tempo, que fosse de baixo custo e envolvesse preferencialmente substâncias de baixa toxicidade. Sendo assim, o principal resultado desse trabalho foi a utilização com sucesso de um método verde que permitiu a obtenção de partículas de ouro muito pequenas com distribuição homogênea que fosse viável econômica e ambientalmente. Sabe-se que para acontecer a formação dessas partículas, os valores dos potenciais padrão de redução $\left(\mathrm{E}^{\circ}\right)$ irão determinar os reagentes necessários para a reação. Isso significa que a alteração da energia livre $\left(\Delta \mathrm{G}^{\circ}\right)$ na reação deve ser negativa (ou que o $\Delta \mathrm{E}^{\circ}>0$ ). Portanto, para que os íons $\mathrm{Au}^{3+}$ sejam reduzidos à $\mathrm{Au}^{0}\left(\mathrm{E}^{\circ}=+1,40 \mathrm{~V}\right)$, torna-se necessário a adição de um agente redutor, no caso, o extrato do limão, que apresenta até quase $7 \%$ de ácido cítrico $\left(\mathrm{E}^{\circ}=-0,180 \mathrm{~V}\right)$. A utilização dos extratos de qualquer um dos tipos de limão usados (taiti, cravo, siciliano, galego) serviu indistintamente tanto para a redução dos íons Au(III) quanto para a estabilização das soluções coloidais, evitando a aglomeração das partículas metálicas. Os procedimentos desenvolvidos substituíram, então, o uso de agentes redutores tóxicos por extratos de diferentes tipos de limão, que quando em altas concentrações, conduziram à formação de partículas muito pequenas da ordem de 5-10 nm. Além disso, as reações são reprodutíveis, processam-se quase que instantaneamente e ocorrem em valores de $\mathrm{pH}$ próximos ao da água, utilizada como solvente (reduzindo, portanto, a quantidade 
a)

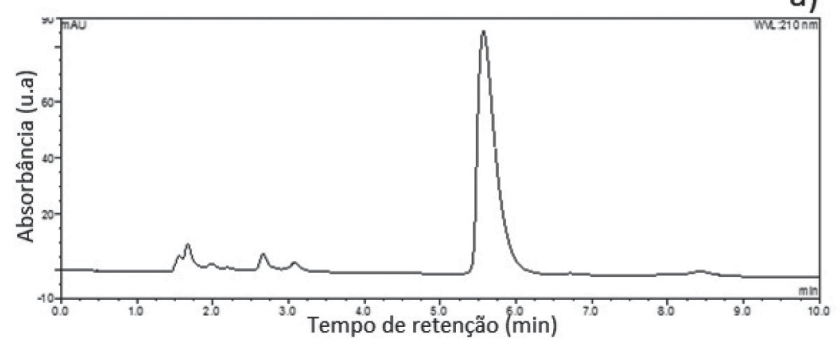

b)

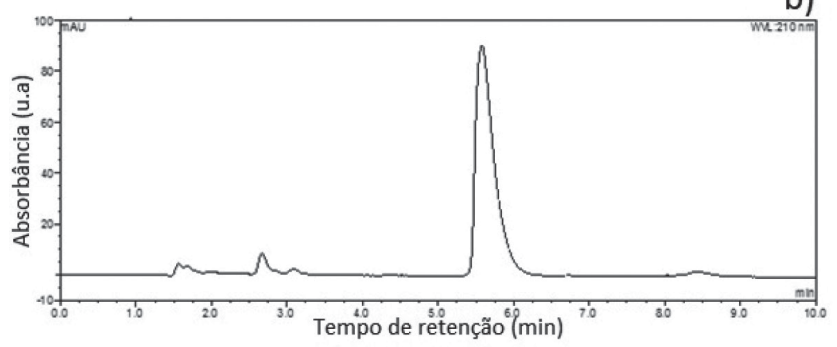

c)

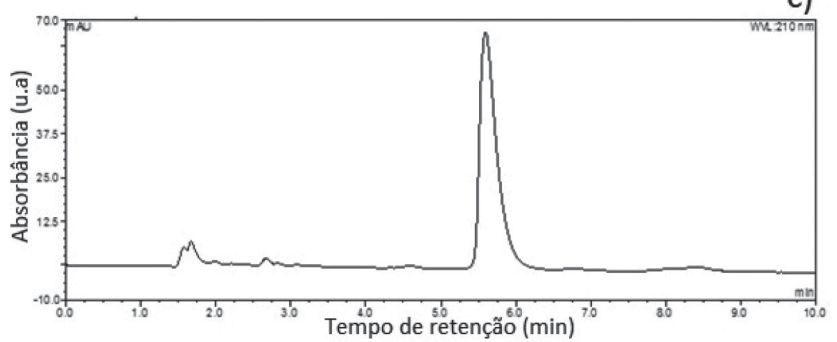

d)

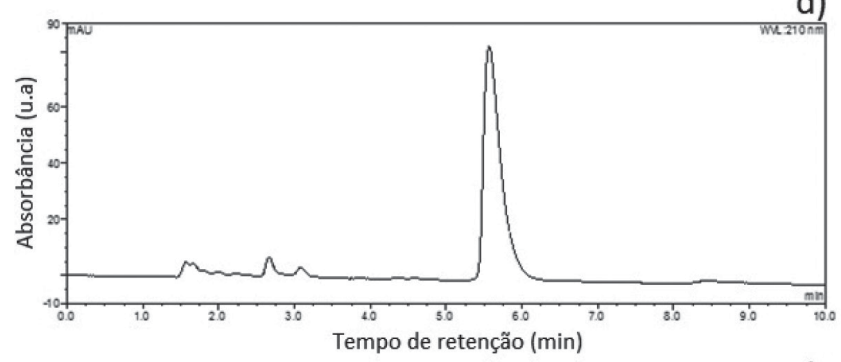

e)

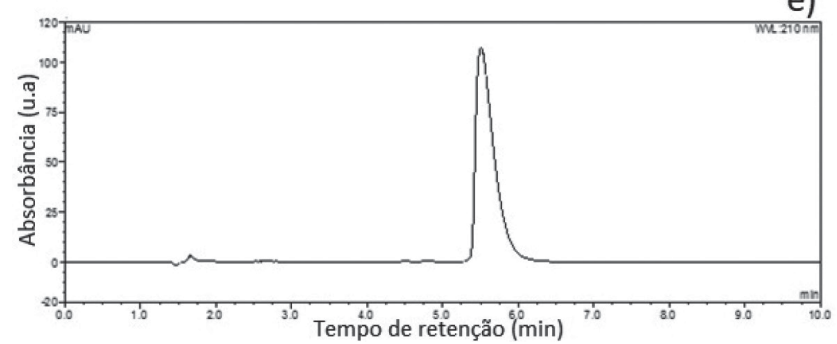

Figura 8. Cromatogramas referentes aos limões a) siciliano, b) taiti, c) cravo, d) galego e e) padrão de ácido cítrico

Tabela 2. Valores obtidos de concentração de ácido cítrico nos limões avaliados

\begin{tabular}{cc}
\hline Tipo limão & Concentração de ácido cítrico $\left(\mathrm{g} \mathrm{L}^{-1}\right)$ \\
\hline Cravo & 47,9 \\
Galego & 59,2 \\
Siciliano & 64,0 \\
Taiti & 69,2 \\
\hline
\end{tabular}

de $\mathrm{NaOH}$ necessária para o ajuste da basicidade do meio). A parte final do trabalho consistiu na produção do precursor ácido cloroáurico a partir da dissolução ácida de um pequeno pedaço de ouro metálico. Essa alternativa apresenta a vantagem de tornar a síntese mais acessível, já que a facilidade de acesso ao ouro metálico é muito maior do que ao ácido cloroáurico. Cabe destacar que os principais resultados apresentados nesse artigo poderão servir não só como subsídios para a obtenção de nanopartículas de ouro em laboratórios de pesquisa de forma rápida, limpa e eficiente, como também para a implantação futura de processos industriais visando a produção em massa desse material.

\section{AGRADECIMENTOS}

Os autores agradecem ao doutorando Tiago Varão do Departamento de Química Analítica pelas análises quantitativas de ouro, ao Laboratório de Microscopia Avançada do Instituto de Química de Araraquara - UNESP (LMA-IQ) pela disponibilidade de utilização dos microscópios eletrônicos e ao CNPq, à FAPESP e à CAPES pelo apoio financeiro.

\section{REFERÊNCIAS}

1. Toma, H. E.; Quim. Nova na Esc. 2005, 21, 3.

2. Drexler, K. E.; Engines of Creation: The Coming Era of Nanotechnology, $1^{\text {st }}$ ed., Anchor Books: New York, 1986.

3. Furley, D. J. Two Studies in the Greek Atomists, $1^{\text {st }}$ ed., Princeton University Press: New Jersey, 1967.

4. Cai, W.; Gao, T.; Hong, H.; Sun, J.; Nanotechnol. Sci. Appl. 2008, 1, 17.

5. Jahangirian, H.; Lemraski, E.; Webster, T.; Rafiee-Moghaddam, R.; Abdollahi, Y.; Int. J. Nanomedicine 2017, 12, 2957.

6. Grassian, V. H.; Haes, A. J.; Mudunkotuwa, I. A.; Demokritou, P.; Kane, A. B.; Murphy, C. J.; Hutchison, J. E.; Isaacs, J. A.; Jun, Y.-S.; Karn, B.; Khondaker, S. I.; Larsen, S. C.; Lau, B. L. T.; Pettibone, J. M.; Sadik, O. A.; Saleh, N. B.; Teague, C.; Environ. Sci. Nano 2016, 3, 15.

7. Murphy, C. J.; Gole, A. M.; Stone, J. W.; Sisco, P. N.; Alkilany, A. M.; Goldsmith, E. C.; Baxter, S. C.; Acc. Chem. Res. 2008, 41, 1721.

8. Tao, A.; Sinsermsuksakul, P.; Yang, P.; Nat. Nanotechnol. 2007, 2, 435.

9. Zaera, F.; Chem. Soc. Rev. 2013, 42, 2746

10. Abdelmohsen, L. K. E. A.; Peng, F.; Tu, Y.; Wilson, D. A.; J. Mater. Chem. B 2014, 2, 2395.

11. Izumi, Y.; Coord. Chem. Rev. 2013, 257, 171.

12. Baffou, G.; Quidant, R.; Chem. Soc. Rev. 2014, 43, 3898.

13. Jain, S.; Hirst, D. G.; O'Sullivan, J. M.; Br. J. Radiol. 2012, 85, 101

14. Bhattacharjee, S.; Brayden, D. J.; Nanomedicine 2015, 10, 2289.

15. McMahon, S. J.; McNamara, A. L.; Schuemann, J.; Prise, K. M.; Paganetti, H.; J. Phys. Conf. Ser. 2017, 777, 12008.

16. Sattler, K. D.; Handbook of Nanophysics: Principles and Methods, $1^{\text {st }}$ ed., CRC Press: Boca Raton, 2010

17. Louis, C.; Pluchery, O.; Gold Nanoparticles for Physics, Chemistry and Biology, $2^{\text {nd }}$ ed., World Scientific: London, 2017.

18. Zhao, H.; Song, F.; Wang, F.; Liu, J.; Liu, Y.; Zhang, J.; Chen, G.; Liu, S.; J Nanosci Nanotechnol 2014, 14, 4357.

19. Savage, A. C.; Pikramenou, Z.; Chem. Commun. 2011, 47, 6431.

20. Daniel, M. C. M.; Astruc, D.; Chem. Rev. 2004, 104, 293.

21. Yue, G.; Su, S.; Li, N.; Shuai, M.; Lai, X.; Astruc, D.; Zhao, P.; Coord. Chem. Rev. 2016, 311, 75.

22. Xu, H.; Li, Y.; Luo, X.; Xu, Z.; Ge, J.; Chem. Commun. 2017, 53, 7953.

23. Zhang, Y.; Cui, X.; Shi, F.; Deng, Y.; Chem. Rev. 2012, 112, 2467.

24. Corma, A.; Garcia, H.; Chem. Soc. Rev. 2008, 37, 2096.

25. Hashmi, A. S. K.; Hutchings, G. J.; Angew. Chem., Int. Ed. 2006, 45, 7896.

26. Zhang, X.; Sun, C.; Fang, N.; J. Nanoparticle Res. 2004, 6, 125. 
27. Mafuné, F.; Kohno, J. Y.; Takeda, Y.; Kondow, T.; J. Phys. Chem. B 2002, 106,7575 .

28. Melo, M. A.; Santos, L. S. S.; Do Carmo Gonçalves, M.; Nogueira, A. F.; Quim. Nova 2012, 35, 1872.

29. Zhao, P.; Li, N.; Astruc, D.; Coord. Chem. Rev. 2013, 257, 638.

30. Zhang, Y.; Chu, W.; Foroushani, A. D.; Wang, H.; Li, D.; Liu, J.; Barrow, C. J.; Wang, X.; Yang, W.; Materials 2014, 7, 5169.

31. Shah, M.; Badwaik, V.; Kherde, Y.; Waghwani, H. K.; Modi, T.; Aguilar, Z. P.; Rodgers, H.; Hamilton, W.; Marutharaj, T.; Webb, C.; Lawrenz, M. B.; Dakshinamurthy, R.; Front. Biosci. 2014, 19, 1320.

32. Da Silva, A. G. M.; Rodrigues, T. S.; Macedo, A.; Da Silva, R. T. P.; Camargo, P. H. C.; Quim. Nova 2014, 37, 1716.

33. Turkevich, J.; Stevenson, P. C.; Hillier, J.; Discuss. Faraday Soc. 1951, 11,55 .

34. Brust, M.; Walker, M.; Bethell, D.; Schiffrin, D. J.; Whyman, R.; J. Chem. Soc., Chem. Commun. 1994, 0, 801.

35. Martin, M. N.; Basham, J. I.; Chando, P.; Eah, S. K.; Langmuir 2010, 26,7410 .

36. Perrault, S. D.; Chan, W. C. W.; J. Am. Chem. Soc. 2010, 132, 11824.

37. Shem, P. M.; Sardar, R.; Shumaker-Parry, J. S.; Langmuir 2009, 25, 13279.

38. Tuchscherer, A.; Schaarschmidt, D.; Schulze, S.; Hietschold, M.; Lang, H.; Inorg. Chem. Commun. 2011, 14, 676.

39. Shan, J.; Tenhu, H.; Chem. Commun. 2007, 4580.

40. Sajid, M.; Ilyas, M.; Basheer, C.; Tariq, M.; Daud, M.; Baig, N.; Shehzad, F.; Environ. Sci. Pollut. Res. 2015, 22, 4122.

41. McGregor, D.; Crit. Rev. Toxicol. 2007, 37, 887.

42. Kimling, J.; Maier, M.; Okenve, B.; Kotaidis, V.; Ballot, H.; Plech, A.; J. Phys. Chem. B 2006, 110, 15700.

43. Da Silva, F. M.; De Lacerda, P. S. B.; Jones Junior, J.; Quim. Nova 2005, $28,103$.

44. Sujitha, M. V.; Kannan, S.; Spectrochim. Acta, Part A 2013, 102, 15.

45. Castro-Longoria, E.; Vilchis-Nestor, A. R.; Avalos-Borja, M.; Colloids Surf., B 2011, 83, 42.

46. Iravani, S.; Acta Naturae 2011, 13, 2638.
47. Yu, J.; Xu, D.; Guan, H. N.; Wang, C.; Huang, L. K.; Chi, D. F.; Mater. Lett. 2016, 166, 110.

48. Qu, Y.; Shen, W.; Pei, X.; Ma, F.; You, S.; Li, S.; Wang, J.; Zhou, J.; J. Environ. Sci. 2017, 56, 79.

49. Zha, J.; Dong, C.; Wang, X.; Zhang, X.; Xiao, X.; Optik 2017, 144, 511.

50. Paul, B.; Bhuyan, B.; Purkayastha, D. D.; Vadivel, S.; Dhar, S. S.; Mater. Lett. 2016, 185, 143.

51. González-Ballesteros, N.; Prado-López, S.; Rodríguez-González, J. B.; Lastra, M.; Colloids Surf., B 2017, 153, 190.

52. http://www.sigmaaldrich.com/technical-documents/articles/materialsscience/nanomaterials/gold-nanoparticles.html, acessada em Fevereiro 2018.

53. www.unicamp.br/nepa/taco/contar/taco_4_edicao_ampliada_e_revisada, acessada em Fevereiro 2018.

54. Couto, M. A. L.; Brazaca, S. G. C.; Ciência e Tecnol. Aliment. 2010, 30, 15.

55. Kimling, J.; Maier, M.; Okenve, B.; Kotaidis, V.; Ballot, H.; Plech, A.; J. Phys. Chem. B 2006, 110, 15700.

56. Goris, B.; Bals, S.; Van Den Broek, W.; Carbó-Argibay, E.; GómezGraña, S.; Liz-Marzán, L. M.; Van Tendeloo, G.; Nat. Mater. 2012, 11, 930.

57. Frem, R. C. G.; Arroyos, G.; Lucena, G. N.; Flor, J. B. S.; Fávaro, M. A.; Coura, M. F.; Alves, R. C. Em Recent Advances in Complex Functional Materials: From Design to Application; Longo, E., La Porta, F. A., eds.; Springer: Cham, 2017; cap. 14.

58. Park, K. S.; Ni, Z.; Côté, A. P.; Choi, J. Y.; Huang, R.; Uribe-Romo, F. J.; Chae, H. K.; O'Keeffe, M.; Yaghi, O. M.; Proc. Natl. Acad. Sci. U. S. A. 2006, 103, 10186.

59. Crupi, M. L.; Costa, R.; Dugo, P.; Dugo, G.; Mondello, L.; Food Chem. 2007, 105, 771.

60. Bruice, P. Y.; Organic Chemistry, $7^{\text {th }}$ ed., Pearson: London, 2012.

61. ANVISA (Agência Nacional de Vigilância Sanitária); Resolução da Diretoria Colegiada - RDC No 166, Guia para validação de métodos analíticos, 2017. 